\title{
Dietary intake of folate equivalents and risk of myocardial infarction in the European Prospective Investigation into Cancer and Nutrition (EPIC)-Potsdam study
}

\author{
Dagmar Drogan ${ }^{1}$, Kerstin Klipstein-Grobusch ${ }^{1, *}{ }^{\dagger}$, Jutta Dierkes $^{2}$, Cornelia Weikert $^{1}$ and \\ Heiner Boeing ${ }^{1}$ \\ ${ }^{1}$ German Institute of Human Nutrition Potsdam-Rehbruecke, Department of Epidemiology, Arthur-Scheunert-Allee \\ 114-116, D-14558 Nuthetal, Germany: ${ }^{2}$ Otto-von-Guericke University, Department of Clinical Chemistry and \\ Pathobiochemistry, Magdeburg, Germany
}

Submitted 4 April 2005: Accepted 10 August 2005

\begin{abstract}
Objective: To assess the relationship between intake of dietary folate equivalents and risk of myocardial infarction in a German cohort.

Design: Intake of dietary folate equivalents was assessed by a validated foodfrequency questionnaire. Cox proportional hazard models were used to evaluate the association between intake of dietary folate equivalents and risk of myocardial infarction.

Setting: The European Prospective Investigation into Cancer and Nutrition (EPIC)Potsdam cohort, Germany.

Subjects: Subjects were 22245 apparently healthy non-users of vitamin supplements aged 35-64 years.

Results: During 4.6 years of follow-up, 129 incident cases of myocardial infarction were identified. Compared with intake below the median $(103 \mu \mathrm{g})$, higher intake of dietary folate equivalents was associated with a multivariate-adjusted hazard ratio (HR) of 0.57 (95\% confidence interval (CI) $0.36-0.91$ ). The inverse association of folate intake and myocardial infarction risk was stronger in participants with an ethanol intake equal to or above the sex-specific median ( $\mathrm{HR}=0.37,95 \% \mathrm{CI}$ $0.18-0.79)$ and attenuated in those with a low ethanol intake (HR $=0.67,95 \% \mathrm{CI}$ $0.37-1.22$ ).

Conclusion: An increased intake of dietary folate equivalents was observed to be associated with decreased risk of myocardial infarction in a German study population, pointing towards the importance of folate intake with respect to primary prevention of myocardial infarction.
\end{abstract}

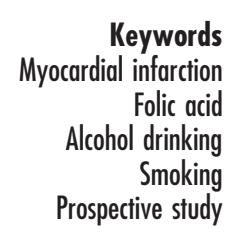

Folate is the generic term that refers to all chemical forms of a water-soluble vitamin with activity similar to that of folic acid (pteroylmonoglutamic acid), the most oxidised and stable compound. The vitamin is involved in the transfer and utilisation of one-carbon units and plays an important role in nucleic acid synthesis and methionine regeneration ${ }^{1}$.

Folate metabolism is closely linked to that of homocysteine. A recent meta-analysis estimated that a $25 \%$ lower usual homocysteine level was significantly associated with an $11 \%$ lower risk of ischaemic heart disease ${ }^{2}$. As dietary folates are one major modifiable determinant of

†Present address: School of Public Health, University of the Witwatersrand, 7 York Road, Parktown 2193, Johannesburg, South Africa. homocysteine ${ }^{3}$, they might also interfere with risk of cardiovascular disease.

However, findings of prospective studies on this issue are inconsistent. Most of them were conducted in North America $^{4-8}$, whereas European data are restricted to one Finnish study ${ }^{9}$. Particularly with regard to current discussions about food fortification in Europe, there is the need for further European analyses about the relationship of dietary folate and major disease risks. Results from American studies can only partly be applied to other populations as food composition, eating habits, proportion of synthetic folic acid in foods and supplements as well as genetic characteristics might differ. Furthermore, folate status may be directly or indirectly influenced by characteristics in lifestyle, such as smoking and alcohol consumption ${ }^{3,10}$. 
In comparison to the present reference value of $400 \mu \mathrm{g}$ dietary folate equivalents (DFE) per day for adults ${ }^{11,12}$, the Western diet often lacks sufficient amounts of the vitamin. As part of the representative German National Health Interview and Examination Survey, current vitamin intakes were measured in the German Nutrition Survey in 1998. For DFE a median dietary intake of $276 \mu \mathrm{g}$ (men) and $229 \mu \mathrm{g}$ (women) was calculated ${ }^{13}$, which changed only slightly after inclusion of data pertaining to supplement use. Given the high prevalence of coronary heart disease worldwide, the reduction of homocysteine by increasing folate intake might be of major public health concern. This is especially true for countries without mandatory food fortification, such as most European countries including Germany.

EPIC-Potsdam is a cohort of the multi-centre European Prospective Investigation into Cancer and Nutrition (EPIC) study. The purpose of the present analysis was to determine whether higher intakes of DFE are associated with a decreased risk of incident fatal and non-fatal myocardial infarction (MI) among 22245 participants of the EPICPotsdam study followed prospectively for 4.6 years and whether such an association differs between subpopulations with a modified MI risk due to factors that may affect folate status (e.g. alcohol consumption and smoking status).

\section{Methods}

\section{Study population}

The EPIC study is a large multi-centre study conducted in 10 European countries. Details of the EPIC-Potsdam cohort, as one part of the German study population, have been published elsewhere ${ }^{14}$. Briefly, women aged 35-64 years and men aged 40-64 years were randomly recruited from the general population. Between August 1994 and September 1998 a total of 27548 subjects (16 644 women and 10904 men) underwent the baseline examination, including a physical examination, self-administered questionnaires on diet and lifestyle, computer-guided interviews and blood sampling. For the current analysis we excluded participants with prevalent ischaemic heart disease at baseline $(n=513)$ and missing dietary $(n=17)$, anthropometric $(n=205)$ or lifestyle $(n=16)$ data. Furthermore, users of vitamin supplements were excluded ( $n=4552$, including 28 cases of incident MI), as potentially protective constituents might distort the results. This left a total of 22245 study participants.

\section{Antbropometry and lifestyle characteristics}

Standardised anthropometric measurements were performed by trained personnel ${ }^{15}$. Body mass index (BMI) was calculated as body weight divided by the square of height $\left(\mathrm{kg} \mathrm{m}^{-2}\right)$. Lifestyle characteristics including regular physical exercise and smoking history were obtained by trained interviewers during a computer-guided interview. Smoking status was expressed as 'present smoking', 'nonsmoking' and 'past smoking'. Physical exercise was defined as the mean time the participants engaged in sporting activities during the summer and winter season (hours per week). For medical history participants answered questions about previous diseases and regular use of medication.

Definition of prevalent hyperlipidaemia was based on self-reports or disease-specific medication, as recorded in the interview. Using oscillometric devices (BOSO-Oscillomat; Bosch \& Sohn, Jungingen, Germany), prevalent hypertension was defined on the basis of the mean between the second and third blood pressure measurement during baseline examination ${ }^{16}$ when the systolic blood pressure was $\geq 140 \mathrm{mmHg}$ and/or diastolic blood pressure was $\geq 90 \mathrm{mmHg}^{17}$. Additionally, prevalent hypertensive and diabetic participants were identified by self-reports, disease-specific medication, or verification by use of medical record or death certificates.

\section{Dietary assessment}

Dietary habits during the previous year were assessed by a self-administered food-frequency questionnaire (FFQ) including questions on frequency and portion size of 148 single food items and regular use of vitamin supplements. Data on validity and reproducibility of the FFQ were reported recently ${ }^{18-21}$. Based on the German food code and nutrient database (BLS 2.3) ${ }^{22}$, nutrient intake was calculated by multiplying the frequency of consumption by nutrient content, dependent on the portion size. To allow for different bioavailability estimates of folate, intake is expressed as 'dietary folate equivalents' (DFE) ${ }^{11,12}$ defined as: $1 \mu \mathrm{g}$ dietary folate equivalent $=1 \mu \mathrm{g}$ dietary folate $=0.5 \mu \mathrm{g}$ synthetic folic acid.

Consumption of alcoholic beverages (i.e. beer, wine, liqueurs and spirits) during the past year was assessed by FFQ. Assessment of alcohol consumption at 20,30 and 40 years of age was done by use of a lifestyle questionnaire. Combining information on both present and past alcohol consumption allowed classification of participants into those who abstained from any alcohol consumption, exdrinkers and those currently consuming alcoholic beverages.

\section{Ascertainment of $\mathrm{MI}$}

Every two to three years participants were asked to answer a mailed follow-up questionnaire that included sections about self-reported MI. Potential incident MI was verified using medical records and/or death certificates, applying criteria based on the World Health Organization MONICA (Monitoring Trends and Determinants in Cardiovascular Disease) study (http://www.ktl.fi/publications/monica/ manual/part4/iv-1.htm). Thus, cases were defined as incident MI according to codes I21.0-I21.9 of the tenth International Classification of Diseases ${ }^{23}$ without previous prevalent ischaemic heart disease occurring between baseline examination and 30 April 2004. 


\section{Statistical analysis}

Statistical analysis was performed using the SAS software package, release 9.1 (SAS Institute, Cary, NC, USA). All tests were performed two-sided with $P<0.05$ considered as statistically significant.

Two categories of DFE intake were calculated for the analytical cohort using median intake as the cut-off point. Baseline characteristics of the study population are expressed as age- and sex-adjusted means and standard error or frequencies, respectively. The statistical significance of differences to be significantly different from zero by category of DFE intake was examined using analysis of covariance.

Follow-up time was computed as the time interval between baseline examination and MI (cases) or between baseline examination and date of death from any cause/drop out/most recent follow-up, whichever came first (remaining cohort). In a Cox regression analysis, age- and sex-adjusted hazard ratios (HR) and corresponding 95\% confidence interval (CI) for the relationship between vitamin DFE intake equal to or above versus below the median and risk of MI were modelled. In the multivariate model we calculated the HR controlling for age, sex, total energy intake (quartiles), BMI (continuous), smoking history (non-smoker, past smoker, present smoker), alcohol consumption at baseline (quartiles), regular exercise $\left(<2 \mathrm{~h} \mathrm{week}^{-1}, \geq 2 \mathrm{~h}\right.$ week $^{-1}$ ), educational attainment (university degree, no university degree), history of diabetes, history of hypertension and history of hyperlipidaemia. The multinutrient HR was further adjusted for intake of dietary fibre, saturated fatty acids and unsaturated fatty acids (all in quartiles). An extended Cox proportional hazard model with counting process style of input was applied using the subject's age at recruitment as the entry time and age at diagnosis of MI or at censoring as the exit time.

To account for possible interactions between DFE and alcohol intake the study population was simultaneously divided according to alcohol consumption, using the sexspecific median as the cut-off point. A further Cox regression analysis was performed estimating the relationship between DFE and risk of MI among subjects with low alcohol consumption and those with an alcohol consumption equal to or above the sex-specific median, respectively. Furthermore, HR of DFE on MI incidence were calculated across present smokers and non-smokers (including ex-smokers).

\section{Results}

\section{Cobort characteristics}

A total of 22245 study participants met the inclusion criteria for the present analysis. During an average followup period of 4.6 years a total of 129 cases of incident MI were identified, of whom 94 ( 73 men and 21 women) were non-fatal and the remaining 35 (25 men and 10 women) were fatal.

In the analytical cohort median DFE intake was $103 \mu \mathrm{g} \mathrm{day}^{-1}$, with DFE mainly deriving from bread, fresh fruits, raw vegetables, beer and dairy products. Selected baseline characteristics of the study participants by median DFE intake are presented in Table 1. Along with

Table 1 Baseline characteristics of 22245 men and women of the EPIC-Potsdam study (1994 to 2004) according to median intake of dietary folate equivalents

\begin{tabular}{|c|c|c|c|}
\hline \multirow[b]{2}{*}{ Variable* $^{*}$} & \multicolumn{3}{|c|}{ Median intake of dietary folate equivalents } \\
\hline & $<103 \mu \mathrm{gday}^{-1}(n=11123)$ & $\geq 103 \mu \mathrm{gday}^{-1}(n=11122)$ & $P$-value \\
\hline Myocardial infarction (\%) & 0.7 & 0.4 & 0.005 \\
\hline Men $(\%) \dagger$ & 27.9 & 51.7 & $<0.0001$ \\
\hline Age (years) $\ddagger$ & $49.8(0.08)$ & $49.4(0.08)$ & 0.001 \\
\hline Body mass index $\left(\mathrm{kg} \mathrm{m}^{-2}\right)$ & $26.3(0.04)$ & $26.3(0.04)$ & 0.684 \\
\hline Current smokers (\%) & 22.4 & 20.0 & $<0.0001$ \\
\hline Regular exercise of $\geq 2$ h week $^{-1}(\%)$ & 11.4 & 14.9 & $<0.0001$ \\
\hline University degree (\%) & 34.9 & 36.4 & 0.018 \\
\hline Hypertension (\%)§ & 47.7 & 48.7 & 0.169 \\
\hline Diabetes (\%) & 0.8 & 0.8 & 0.779 \\
\hline Hyperlipidaemia (\%)\| & 27.8 & 26.9 & 0.151 \\
\hline Dietary vitamin $\mathrm{B}_{6}\left(\mathrm{mg}\right.$ day $\left.^{-1}\right)$ & $1.3(0.003)$ & $1.9(0.003)$ & $<0.0001$ \\
\hline Dietary vitamin $B_{12}\left(\mu \mathrm{g}\right.$ day $\left.^{-1}\right)$ & $5.3(0.02)$ & $7.8(0.02)$ & $<0.0001$ \\
\hline Dietary fibre $\left(\mathrm{g} \mathrm{day}^{-1}\right)$ & $18.3(0.05)$ & $26.1(0.05)$ & $<0.0001$ \\
\hline Saturated fatty acids intake $\left(\mathrm{g} \mathrm{day}^{-1}\right)$ & $28.5(0.12)$ & $40.1(0.12)$ & $<0.0001$ \\
\hline Polyunsaturated fatty acids intake (g day ${ }^{-1}$ ) & $12.5(0.05)$ & $16.9(0.05)$ & $<0.0001$ \\
\hline Ethanol intake $\left(\mathrm{g} \mathrm{day}^{-1}\right)$ & $11.0(0.17)$ & $17.6(0.17)$ & $<0.0001$ \\
\hline Energy intake (kJ day $\left.{ }^{-1}\right)$ & $7332(21)$ & $10411(21)$ & $<0.0001$ \\
\hline
\end{tabular}

EPIC - European Prospective Investigation into Cancer and Nutrition.

*Values are age- and sex-adjusted means (standard error) or \% of participants as indicated.

† Adjusted for age only.

$\ddagger$ Adjusted for sex only.

$\S$ Systolic blood pressure $\geq 140 \mathrm{mmHg}$ and/or diastolic blood pressure $\geq 90 \mathrm{mmHg}$ and/or use of antihypertensive medication and/or self-reports and/or medical verification during follow-up.

I Disease-specific medication and/or self-reports and/or medical verification during follow-up.

|| Disease-specific medication and/or self-reports. 
differences in sex distribution there were also differences in the proportion of cases, current smokers, participants with higher educational attainment and participants with regular sporting activities. A lower DFE intake was associated with lower intake of total energy and thus with lower intake of other dietary components. After adjustment for age, sex and intake of total energy, mean intakes of ethanol, dietary fibre and vitamins $\mathrm{B}_{6}$ and $\mathrm{B}_{12}$ were still significantly lower in participants with DFE intake below the median (data not shown).

\section{Intake of DFE and risk of MI}

DFE intake equal to or above the median $\left(103 \mu \mathrm{g} \mathrm{day}^{-1}\right)$ was reported by $46.5 \%$ of cases and $50.0 \%$ of the remaining cohort. Higher amounts of DFE intake significantly decreased the risk of MI by more than $30 \%$, depending on the covariates included in the model (Table 2). In a Cox proportional hazards model adjusted for age and sex, study participants of the higher-folate diet had a relative MI risk of 0.63 (95\% CI $0.44-0.90)$ compared with the low-folate diet. In the multivariate model further adjusted for total energy intake, BMI, smoking history, alcohol consumption, regular exercise, educational attainment and history of diabetes/hypertension/hyperlipidaemia, an HR of 0.52 (95\% CI $0.33-0.81$ ) was calculated. Further adjustment for intakes of fibre, saturated fatty acids and unsaturated fatty acids (multinutrient model) only slightly altered the HR to 0.57 (95\% CI 0.36-0.91). The significant inverse association between DFE and MI risk persisted when daily intakes of vitamin $\mathrm{A}, \beta$-carotene, vitamin $\mathrm{C}$ and vitamin $\mathrm{E}$ were included (data not shown). In separate analyses for nonfatal and fatal MI, similar HR were observed (data not shown). For non-fatal cases this association was always statistically significant. Due to the small number of fatal cases $(n=35)$ confidence limits were broader, thus leading to non-significant results.

\section{Joint associations of DFE intake with alcohol consumption or smoking status on MI risk}

Among subgroups additional analyses were conducted to examine the association between intake of DFE and MI risk in combination with factors that may modulate folate or homocysteine levels.

Based on the median DFE the cohort was further divided according to sex-specific median alcohol consumption. Daily ethanol intake below the median (men: $17.4 \mathrm{~g}$, women: $5.1 \mathrm{~g}$ ) was reported by $61 \%$ of cases with incident MI and 50\% of control subjects. In this stratum, a higher intake of DFE (30 cases) was associated with a non-significantly decreased HR of 0.67 (95\% CI $0.37-1.22)$ in the multinutrient-adjusted model, as illustrated in Fig. 1. In contrast, DFE intake equal to or above the median (30 cases) was associated with a 60\% decreased risk ( $\mathrm{HR}=0.37,95 \%$ CI $0.18-0.79)$ in participants with an alcohol consumption above the sexspecific median.

Because alcohol is often given up due to health problems, present abstainers might have a higher risk profile for MI. Exclusion of participants reporting past alcohol consumption but no alcohol consumption at baseline (529 participants including nine cases of incident MI) only slightly altered the sex-specific median ethanol intake (men: $18.2 \mathrm{~g} \mathrm{day}^{-1}$, women: $5.3 \mathrm{~g} \mathrm{day}^{-1}$ ) and risk estimates. In comparison to the reference group of low DFE, the inverse association between DFE and MI risk was strongest among those with an ethanol intake equal to or above the sex-specific median ( $\mathrm{HR}=0.39$, 95\% CI $0.18-$ 0.83), whereas no significant risk reduction by DFE was detected in those with an ethanol intake below the median $(\mathrm{HR}=0.65,95 \%$ CI $0.34-1.24)$.

We additionally assessed the joint effects of DFE intake and smoking status on MI risk as current smoking might interfere with folate and homocysteine status. Median daily intake of DFE was $102 \mu \mathrm{g}$ among smokers and $103 \mu \mathrm{g}$

Table 2 Hazard ratios (HR) (95\% confidence interval $(\mathrm{Cl})$ ) of myocardial infarction according to median intake of dietary folate equivalents in 22245 men and women of the EPIC-Potsdam study (1994 to 2004)

\begin{tabular}{lccc}
\hline & \multicolumn{3}{c}{ Intake of dietary folate equivalents } \\
\cline { 2 - 4 } & $<103 \mu$ gday $^{-1}$ & $\geq 103 \mu$ g day $^{-1}$ & $P$-value \\
\hline Study participants $(n)$ & 11123 & 11122 & \\
Person-years & 51540 & 51620 & \\
Cases of myocardial infarction $(n)$ & 69 & 60 & 0.01 \\
HR (95\% Cl) & 1.00 & $0.63(0.44-0.90)$ & 0.004 \\
Age- and sex-adjusted & 1.00 & $0.52(0.33-0.81)$ & 0.018 \\
Multivariate adjusted* & 1.00 & $0.57(0.36-0.91)$ & 0.018 \\
\hline Multinutrient adjusted $\dagger$ & & & \\
\hline
\end{tabular}

EPIC - European Prospective Investigation into Cancer and Nutrition.

* Multivariate model: adjusted for age, sex, total energy intake (quartiles), body mass index, smoking history (non-smoker, past smoker, present smoker), alcohol consumption (quartiles), regular exercise $\left(<2 \mathrm{hweek}^{-1}, \geq 2 \mathrm{hweek}^{-1}\right)$, educational attainment (university degree, no university degree), history of diabetes, history of hypertension and history of hyperlipidaemia. † Multinutrient model: multivariate model further adjusted for daily intake of fibre, saturated fatty acids and polyunsaturated fatty acids (all quartiles). 


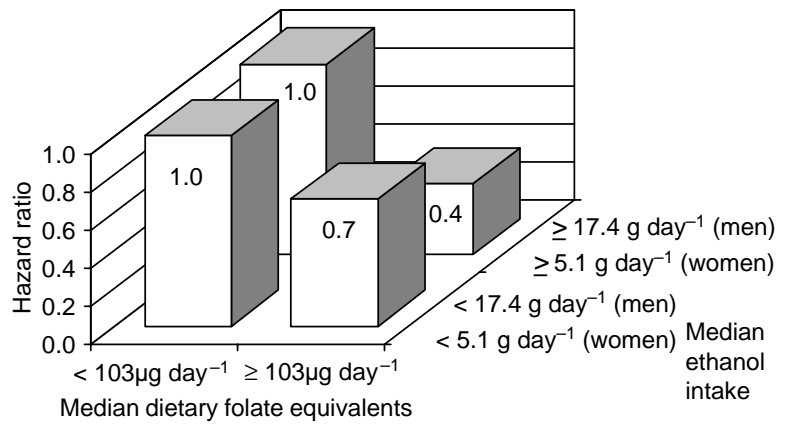

Fig. 1 Hazard ratios ${ }^{*}$ of myocardial infarction according to median intake of dietary folate equivalents by sex-specific median ethanol intake in 22245 men and women of the EPIC-Potsdam study (1994 to 2004). Reference category was dietary folate equivalent intake below the median. *Adjusted for age, sex, total energy intake (quartiles), body mass index, smoking history (non-smoker,

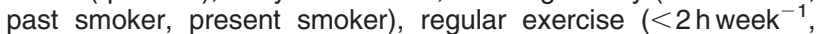
$\geq 2 \mathrm{hweek}^{-1}$ ), educational attainment (university degree, no university degree), history of diabetes, history of hypertension, history of hyperlipidaemia, daily intake of fibre (quartiles), daily intake of saturated fatty acids (quartiles) and daily intake of polyunsaturated fatty acids (quartiles). EPIC - European Prospective Investigation into Cancer and Nutrition

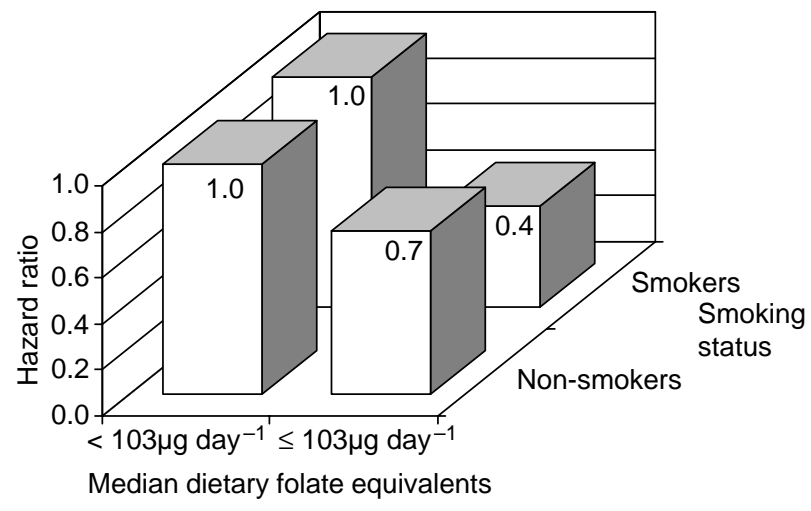

Fig. 2 Hazard ratios* of myocardial infarction according to median intake of dietary folate equivalents by smoking status in 22245 men and women of the EPIC-Potsdam study (1994 to 2004). Reference category was dietary folate equivalent intake below the median. *Adjusted for age, sex, total energy intake (quartiles), body mass index, alcohol consumption (quartiles), regular exercise $\left(<2 \mathrm{~h}\right.$ week $^{-1}, \geq 2 \mathrm{~h}$ week $^{-1}$ ), educational attainment (university degree, no university degree), history of diabetes, history of hypertension, history of hyperlipidaemia, daily intake of fibre (quartiles), daily intake of saturated fatty acids (quartiles) and daily intake of polyunsaturated fatty acids (quartiles). EPIC European Prospective Investigation into Cancer and Nutrition

among non-smokers and did not differ significantly between both groups. Of the 4710 present smokers, a total of 2370 including $36 \mathrm{MI}$ cases reported a DFE intake of less than $103 \mu \mathrm{g} \mathrm{day}^{-1}$. As illustrated in Fig. 2, DFE intake equal to or above the median was associated with a significant risk reduction by more than $50 \%$ in the multinutrient-adjusted model ( $\mathrm{HR}=0.44,95 \%$ CI $0.23-0.87)$ among currently smoking participants. For non-smokers, a higher DFE intake was associated with a non-significant reduction in MI incidence ( $\mathrm{HR}=0.71,95 \% \mathrm{CI} 0.37-1.38)$.

\section{Discussion}

In the current analysis of the EPIC-Potsdam study the risk of incident MI decreased significantly with higher intake of DFE. Although the small number of cases limited the possibility for a detailed dose-response analysis, a more than 30\% reduction in MI incidence for an intake of DFE equal to or above the median $\left(103 \mu \mathrm{g} \mathrm{day}^{-1}\right)$ has been observed after adjustment for traditional coronary risk factors and nutrient intake.

There is a tight metabolic interaction between folate and homocysteine. Although stronger associations were observed in retrospective studies than in prospective studies, elevated levels of homocysteine have been suggested to be an independent risk factor for cardiovascular disease ${ }^{2}$. Higher intakes of dietary folates might reduce risk of cardiovascular disease as they are associated with decreased blood levels of homocysteine ${ }^{3}$.

So far, the importance of dietary folate for cardiovascular disease has been assessed only by a few cohort studies, predominantly conducted on North American populations. However, findings were inconsistent and a protective effect of the vitamin was often limited to the upper intake category which included a high proportion of participants using vitamin supplements or consuming food fortified with folic acid.

In the Nurses' Health Study, 658 incident cases of coronary heart disease were documented during 14 years of follow-up. Dietary data were obtained by use of an FFQ. There was a significant decrease in risk (relative risk $(\mathrm{RR})=0.69,95 \%$ CI $0.55-0.87)$ for women in the highest quintile of folate intake (median intake $696 \mu \mathrm{g} \mathrm{day}^{-1}$ ) compared with those in the lowest (median intake $\left.158 \mu \mathrm{g} \mathrm{day}^{-1}\right)^{6}$. More recently, after an average followup period of 16 years, Jiang et al. ${ }^{8}$ reported a negative association between total folate intake and risk of cardiovascular disease $(n=2381)$ in women when comparing participants with an intake below $180 \mu \mathrm{g} \mathrm{day}^{-1}$ and those with an intake of 400$599 \mu \mathrm{g} \mathrm{day}^{-1}$ ( $\mathrm{RR}=1.25,95 \%$ CI $\left.1.05-1.48\right)$.

After an average of 19 years of follow-up in NHANES I (National Health and Nutrition Examination Survey), Bazzano et $\mathrm{al}^{7}$ analysed 3758 incident cases of cardiovascular disease. For the comparison between extreme quartiles of dietary folate, based on a single 24hour dietary recall, an RR of 0.87 (95\% CI 0.79-0.96) was calculated. In contrast, no relationship between dietary folate, assessed by a 24-hour-recall, and fatal coronary heart disease $(n=165)$ was observed in the Nutrition Canada Survey cohort after 15 years of followup $^{4}$. In an analysis of the ARIC (Atherosclerosis Risk in Communities) study mean intake of dietary folate did not differ significantly $(P=0.78)$ between 232 cases of incident coronary heart disease and a random sample of 527 participants of the cohort after 3.3 years of follow-up 5 . 
To the best of our knowledge, the Kuopio Ischemic Heart Disease Risk Factor Study is the only European study which has investigated this topic so far ${ }^{9}$. In this exclusively male population, the highest fifth of folate intake, assessed by a 4-day food record, was associated with a significantly decreased risk of acute coronary events $(R R=0.45,95 \%$ CI 0.25-0.81).

Comparisons to the present analysis may be limited because of the relatively low proportion of enriched foods in Germany and characteristics of the EPIC-Potsdam cohort with respect to genetics and lifestyle habits that might modulate folate status. Furthermore, generalisations to the present analysis are difficult due to differences in terms of cardiovascular endpoints, dietary assessment instruments as well as the distribution between the sexes.

An excess consumption of alcohol is known to diminish folate status by intestinal malabsorption, decreased hepatic uptake, increased renal excretion of the vitamin and interference with hepatic methionine metabolism ${ }^{10}$. On the other hand, moderate alcohol consumption is protective for cardiovascular disease risk on its own. In contrast to chronic alcoholism, the interaction between folate status and low or moderate alcohol consumption is less well understood. Therefore, the joint association of DFE and alcohol intake with risk of MI was investigated in the present study. The notable inverse relationship between dietary folate and MI risk among participants of moderate or higher ethanol intake is in concordance with an analysis of the Nurses' Health Study, where the risk reduction of coronary heart disease associated with high dietary folate intake was strongest among women consuming alcoholic beverages compared with non-drinkers ${ }^{6}$.

A problem in investigating alcohol and MI risk is that current abstainers might have given up drinking recently due to health problems and therefore lead to misclassification. However, exclusion of participants who stated exdrinking resulted in similar risk estimates in our analysis. In a cross-sectional study Koehler et al. found a stronger negative association between food folate intake and predicted serum homocysteine in alcohol users than in non-users ${ }^{24}$. Although not explained so far, this might also be the underlying mechanism for the strengthened beneficial effect of folate intake in participants with a higher ethanol intake in our study.

Smoking is one of the classical risk factors for $\mathrm{MI}^{25}$. Several studies have demonstrated a positive association between smoking and homocysteine, which even persisted after adjustment for folate intake ${ }^{3}$. In order to examine whether smoking status might thus modify the positive association between DFE and MI risk, stratified analyses were conducted among smokers and nonsmokers. We found the beneficial effects of higher dietary folate to be stronger and statistically significant in currently smoking participants. Thus, our results do not support the finding of the Kuopio Ischemic Heart Disease Risk Factor Study that an inverse association between dietary folate and risk of acute coronary events existed primarily in nonsmokers and was rather weak in smokers?

There are some methodological aspects related to our study that should be considered, such as the relatively short follow-up period resulting in a small number of MI cases. Thus, minor changes in risk estimates might not have reached statistical significance. To reduce the magnitude of this effect, participants were divided according to their median intake data into two categories only. Therefore, each category contained a reasonable number of cases and controls for statistical modelling.

Intake of DFE was calculated on the basis of a nutrient database $^{22}$ that might be subject to inaccuracies due to changes in the folate content of foods, resulting in misclassification of participants. Additionally, intake data might not sufficiently reflect differences in bioavailability of dietary folates as well as specific storage conditions and preparation methods. Because the FFQ is a semiquantitative assessment instrument, it might be subject to misclassification, too. Validity and reproducibility of the questionnaire for DFE intake have so far not been assessed. However, intake of food groups that are major DFE sources in the EPIC-Potsdam study was found to be reproducible and of moderate validity ${ }^{18}$. Spearman correlation coefficients between intake values derived from 12 24-hour diet recalls and the FFQ were 0.51 for bread, 0.50 for fruits, 0.34 for vegetables and 0.56 for milk and milk products.

Dividing the study population into two categories only takes into account possible inaccuracy in estimating folate intake. Furthermore, random errors in measurement tend to bias any association towards the null rather than create significant risk estimates. Even if subjects changed their dietary habits during follow-up due to health problems possibly associated with MI risk, a weakened or nonsignificant association would be expected.

One major strength of the current study is its prospective character. We tried to minimise possible confounding by considering important dietary and non-dietary variables in the Cox model associated with healthy lifestyle practices or a certain MI risk profile. Adjustment for these factors only slightly altered risk estimates in comparison to the age- and sex-adjusted model. Furthermore, users of vitamin supplements have been excluded from the present analysis as supplement use has been associated with certain lifestyle characteristics in the EPIC-Potsdam cohort that might also modify MI risk estimates ${ }^{26}$. As our data do not allow correct quantification of folic acid intake from supplements, exclusion of those subjects consuming supplements reduced misclassification.

In conclusion, a higher intake of DFE was associated with a substantially lower risk of fatal and non-fatal MI in this German population of middle-aged men and women. Assuming a causal influence of dietary folates on MI risk and given the high incidence rates of MI, increasing folate intake should be a major public health concern. 


\section{Acknowledgements}

The present study was supported by the Bundesministerium für Bilding und Forschung (research grant no. 0312750B). We thank Ellen Kohlsdorf and Wolfgang Bernigau for data management, Wolfgang Fleischhauer for ascertainment of MI cases and Kurt Hoffmann for statistical support.

\section{References}

1 Scott JM, Weir DG. Folic acid, homocysteine and one-carbon metabolism: a review of the essential biochemistry. Journal of Cardiovascular Risk 1998; 5: 223-7.

2 Homocysteine Studies Collaboration. Homocysteine and risk of ischemic heart disease and stroke: a meta-analysis. Journal of the American Medical Association 2002; 288: 2015-22.

3 De Bree A, Verschuren WM, Kromhout D, Kluijtmans LA, Blom HJ. Homocysteine determinants and the evidence to what extent homocysteine determines the risk of coronary heart disease. Pharmacological Reviews 2002; 54: 599-618.

4 Morrison HI, Schaubel D, Desmeules M, Wigle DT. Serum folate and risk of fatal coronary heart disease. Journal of the American Medical Association 1996; 275: 1893-6.

5 Folsom AR, Nieto FJ, McGovern PG, Tsai MY, Malinow MR, Eckfeldt JH, et al. Prospective study of coronary heart disease incidence in relation to fasting total homocysteine, related genetic polymorphisms, and $B$ vitamins: the Atherosclerosis Risk in Communities (ARIC) study. Circulation 1998; 98: 204-10.

6 Rimm EB, Willett WC, Hu FB, Sampson L, Colditz GA, Manson JE, et al. Folate and vitamin $\mathrm{B}_{6}$ from diet and supplements in relation to risk of coronary heart disease among women. Journal of the American Medical Association 1998; 279: 359-64.

7 Bazzano LA, He J, Ogden LG, Loria C, Vupputuri S, Myers L, et al. Dietary intake of folate and risk of stroke in US men and women: NHANES I Epidemiologic Follow-up Study. National Health and Nutrition Examination Survey. Stroke 2002; 33: 1183-8.

8 Jiang R, Hu FB, Giovannucci EL, Rimm EB, Stampfer MJ, Spiegelman D, et al. Joint association of alcohol and folate intake with risk of major chronic disease in women. American Journal of Epidemiology 2003; 158: 760-71.

9 Voutilainen S, Rissanen TH, Virtanen J, Lakka TA, Salonen JT. Low dietary folate intake is associated with an excess incidence of acute coronary events: The Kuopio Ischemic Heart Disease Risk Factor Study. Circulation 2001; 103: 2674-80.

10 Halsted CH, Villanueva JA, Devlin AM, Chandler CJ. Metabolic interactions of alcohol and folate. Journal of Nutrition 2002; 132: 2367S-72S.

11 Deutsche Gesellschaft für Ernährung. Referenzwerte für die Näbrstoffzufubr. Frankfurt am Main: Umschau/Braus, 2000.

12 Food and Nutrition Board, Institute of Medicine. Dietary Reference Intakes: Thiamin, Riboflavin, Niacin, Vitamin $B_{6}$, Folate, Vitamin $B_{12}$, Pantothenic Acid, Biotin, and Choline. Washington, DC: National Academy Press, 1998.

13 Beitz R, Mensink GB, Fischer B, Thamm M. Vitamins - dietary intake and intake from dietary supplements in Germany. European Journal of Clinical Nutrition 2002; 56: 539-45.
14 Boeing H, Korfmann A, Bergmann MM. Recruitment procedures of EPIC-Germany. European Investigation into Cancer and Nutrition. Annals of Nutrition \& Metabolism 1999; 43: 205-15.

15 Klipstein-Grobusch K, Georg T, Boeing H. Interviewer variability in anthropometric measurements and estimates of body composition. International Journal of Epidemiology 1997; 26(Suppl. 1): S174-80.

16 Schulze MB, Kroke A, Bergmann MM, Boeing H. Differences of blood pressure estimates between consecutive measurements on one occasion: implications for inter-study comparability of epidemiologic studies. European Journal of Epidemiology 2000; 16: 891-8.

17 Chalmers J, MacMahon S, Mancia G, Whitworth J, Beilin L, Hansson L, et al. World Health Organization-International Society of Hypertension guidelines for the management of hypertension. Guidelines sub-committee of the World Health Organization. Clinical and Experimental Hypertension 1999; 21: 1009-60.

18 Bohlscheid-Thomas S, Hoting I, Boeing H, Wahrendorf J. Reproducibility and relative validity of food group intake in a food frequency questionnaire developed for the German part of the EPIC project. European Prospective Investigation into Cancer and Nutrition. International Journal of Epidemiology 1997; 26: S59-70.

19 Bohlscheid-Thomas S, Hoting I, Boeing H, Wahrendorf J. Reproducibility and relative validity of energy and macronutrient intake of a food frequency questionnaire developed for the German part of the EPIC project. European Prospective Investigation into Cancer and Nutrition. International Journal of Epidemiology 1997; 26: S71-81.

20 Boeing H, Bohlscheid-Thomas S, Voss S, Schneeweiss S, Wahrendorf $J$. The relative validity of vitamin intakes derived from a food frequency questionnaire compared to 24-hour recalls and biological measurements: results from the EPIC pilot study in Germany. European Prospective Investigation into Cancer and Nutrition. International Journal of Epidemiology 1997; 26: S82-90.

21 Kroke A, Bergmann MM, Lotze G, Jeckel A, KlipsteinGrobusch K, Boeing H. Measures of quality control in the German component of the EPIC study. European Prospective Investigation into Cancer and Nutrition. Annals of Nutrition \& Metabolism 1999; 43: 216-24.

22 Dehne LI, Klemm C, Henseler G, Hermann-Kunz E. The German food code and nutrient data base (BLS II.2). European Journal of Epidemiology 1999; 15: 355-9.

23 World Health Organization (WHO). International Statistical Classification of Diseases and Related Health Problems. Geneva: WHO, 1992.

24 Koehler KM, Baumgartner RN, Garry PJ, Allen RH, Stabler SP, Rimm EB. Association of folate intake and serum homocysteine in elderly persons according to vitamin supplementation and alcohol use. American Journal of Clinical Nutrition 2001; 73: 628-37.

25 Grundy SM, Pasternak R, Greenland P, Smith S Jr, Fuster V. Assessment of cardiovascular risk by use of multiple-risk-factor assessment equations: a statement for healthcare professionals from the American Heart Association and the American College of Cardiology. Circulation 1999; 100: 1481-92.

26 Klipstein-Grobusch K, Kroke A, Voss S, Boeing H. [Influence of lifestyle on the use of supplements in the Brandenburg nutrition and cancer study]. Zeitschrift fur Ernabrungswissenschaft 1998; 37: 38-46. 